\title{
Relationship between Menstrual Profile and Psychological Stress with Dysmenorrhea
}

\author{
Maryam, ${ }^{1}$ Mulyanusa Amarullah Ritonga, ${ }^{2}$ Istriati $^{3}$ \\ ${ }^{1}$ Faculty of Medicine Universitas Padjadjaran, ${ }^{2}$ Department of Obstetrics and Gynaecology Faculty \\ of Medicine Universitas Padjadjaran/Dr. Hasan Sadikin General Hospital Bandung, ${ }^{3}$ Department \\ of Pharmacology and Therapy Faculty of Medicine Universitas Padjadjaran
}

\begin{abstract}
Background: Dysmenorrhea or menstrual cramps is the most common menstrual complaint among reproductive women in developing countries. The prevalence of dysmenorrhea is ranged from 19-91\%. Many studies showed that factors associated with dysmenorrhea include a younger age, nulliparity, family history of dysmenorrhea, prolonged menstrual duration, high menstrual flow, psychological factors, and lifestyle. The objective of this study was to compare female medical students with dysmenorrhea and without dysmenorrhea regarding menstrual profile and psychological stress, also to examine the relationship between psychological stress and dysmenorrhea specifically..

Methods: This cross-sectional study was conducted among 136 fourth year female medical students of Universitas Padjadjaran. It used a structured, self-assessment, validated, and reliable questionnaire, such as Wong Baker Faces® Pain Rating Scale in combination with Numeric Scale Pain, and Depression, Anxiety, Stress Scale (DASS-42). Then, the descriptive data analysis and chi-square hypothesis test were used to explore the relationship between the severity of psychological stress and the presence or severity of dysmenorrhea.
\end{abstract}

Results: Family history of dysmenorrhea had a strongest relationship with dysmenorrhea. Nevertheless, there was no statistically significant interaction between the severity of stress and the presence of last menstrual cramps (p 0.745, Odd Ratio 1.15, 95\% Confidence Interval 0.5-2.66). However, a positive relationship between the severity of stress and dysmenorrhea in last menstruation was found in this study (p 0.033, Odd Ratio 3.69, 95\% Confidence Interval 1.06-12.8).

Conclusions: The severity of stress and dysmenorrhea in last menstruation is related among the subjects with dysmenorrhea. [AMJ.2016;3(3):382-7]

Keywords: Dysmenorrhea, medical students, menstrual profile, psychological stress

\section{Introduction}

Dysmenorrhea which is defined as a pain in lower abdomen and often accompanied by other symptoms occurring during menstruation, is the most common menstrual complaint among reproductive women in developing countries compared to amenorrhea, polymenorrhea, and menorrhagia., ${ }^{1,2}$ The prevalence of dysmenorrhea is ranged from $19-91 \% 0^{3-5}$ Its varieties may be caused by different pain perception and lack of standard methods to define dysmenorrhea. ${ }^{3}$ Dysmenorrhea can cause economic losses due to the cost of medications, medical care, and decreased productivity. ${ }^{1}$ Previous study in 2009 among female medical students in Malaysia ${ }^{6}$ showed that the prevalence of dysmenorrhea reaches $52.2 \%$ of 235 subjects with their mean age
21.4 years old. Disability among students with dysmenorrhea is also worse in aspect of social activities, responsibilities, time spent resting, class absenteeism, and normal daily life compared to students without dysmenorrhea. ${ }^{6}$

Dysmenorrhea may be viewed as a multifactorial condition. Many studies showed that factors associated with dysmenorrhea include a younger age, nulliparity, family history of dysmenorrhea, prolonged menstrual duration, high menstrual flow, psychology, and lifestyle such as smoking habits, high alcohol, and caffeine intake., 3 So far, few studies associating with psychological stress and dysmenorrhea have been carried out.

The objective of this study was to compare female medical students with dysmenorrhea and without dysmenorrhea regarding menstrual characteristics, gynecological 
problems, family history of dysmenorrhea, lifestyle, and psychological stress, specifically to examine the relationship between psychological stress and dysmenorrhea.

\section{Methods}

This cross-sectional study was conducted in September 2015 using total sampling among 136 fourth year female medical students of Universitas Padjadjaran Bandung, West Java, aged 19-22 years old who had agreed to participate in this study and gave their consent. The subject who did not fill the questionnaire completely was excluded from this study.

The ethical clearance was obtained from the Health Research Ethic Committee Faculty of Medicine Universitas Padjadjaran. The distribution was then taking place at skills lab during class break.

Primary data from a structured, selfassessment, validated, and reliable questionnaire was used for this study. Its questions consisted of psychological stress condition as an independent variable; pain scale of dysmenorrhea as a dependent variable; and other characteristics that might be considered as confounding variables such as age; gynecologic factors including regularity, cycle, duration, amount of bleeding, presence of gynecologic problems, and family history of dysmenorrhea; and lifestyle including smoking habit, alcohol and coffee consumption.

Depression anxiety stress scale (DASS-42) that has been translated into Bahasa Indonesia $(\alpha=0.95)$ was used for measuring psychological stress. This questionnaire consisted of 42 questions including 14 questions to measure a range of common symptoms of stress subscale $(\alpha=0.88)$. The scores were on a four-point Likert scale scored from zero to three. Therefore, the total score of stress subscale ranges from 0 to 42. The cut-off for stress severity subscale was considered as follows, $0-18$, no-mild stress; $>19$, moderate-severe stress. ${ }^{8}$

Combination of Wong-Baker faces ${ }^{\circledR}$ pain rating scale and numeric pain rating scale was used to measure the severity of dysmenorrhea on last menstruation. It consists of six different facial expressions, from a very smiling face to a very sad face, in combination with elevenpoint scale scored from zero to ten, indicating no pain to worst pain intensities. Cut-off 0 for no dysmenorrhea, 1-3 for mild dysmenorrhea, 4-6 for moderate dysmenorrhea, and 7-10 for severe dysmenorrhea were used in this study. ${ }^{9,10}$

A structured questionnaire used to assess other characteristics that may be considered as confounding variables, consists of 'demographic factors' including age, 'gynecologic factors' such as regularity, cycle, duration, amount of bleeding, presence of gynecologic problems, and family history of dysmenorrhea; the regularity of each menstrual cycle was considered as regular menstruation. The menstrual cycle was classified into $<25$ days, $25-32$ days, and $>32$ days. The menstrual duration was classified into $<7$ days, $7-14$ days, and $>14$ days. The amount of bleeding was classified into $<3$ pads/day, 3-7 pads/day, and $>8$ pads/day. The presence of gynecological pathology such as endometriosis and ovarian cyst that have been diagnosed by gynecologist was considered as positive gynecological problems. The presence of dysmenorrhea in student's sister or mother was considered as a positive family history of dysmenorrhea. In addition, the 'lifestyle factors' including daily smoking habit, alcohol and coffee intake were also assessed.

The collected data were statistically using analyzed chi-square test A p-value $<0.05$ that was considered statistically significant, meanwhile odd ratio and 95\% confidence intervals were presented for the main association

\section{Results}

The overall incidence of dysmenorrhea in last menstruation was 74 subjects $(54.4 \%)$ with the mild, moderate, and severe dysmenorrhea that reached $9(12.1 \%), 27(36.5 \%)$, and 38 $(51.4 \%)$, respectively. The average age was 20.57 years old; it was similar to women with dysmenorrhea and without dysmenorrhea. The general characteristics of subjects are shown in the Table 1.

Irregular menstrual cycle was reported by 30 subjects (22\%) and $60 \%$ of those was having dysmenorrhea on their last menstruation. Menstrual cycle was <25 days in 14 subjects $(10 \%), 25-32$ days in 105 subjects $(77 \%)$, and $>25$ days in 17 subjects $(13 \%)$. Menstrual duration was $<7$ days in 73 subjects $(54 \%)$ and $56 \%$ of those was having dysmenorrhea on their last menstruation. The amount of bleeding was assessed according to pads usage in a day, there were $<3$ pads/day in 58 subjects $(43 \%)$ and $3-8$ pads/day in 78 subjects $(57 \%)$. There were only 5 subjects $(4 \%)$ who had any gynecological problems such as endometriosis and ovarian cyst. In this study, there was no statistically significant characteristic between dysmenorrhea and non-dysmenorrhea group, 
Table 1 General Characteristics of the Study Population

\begin{tabular}{|c|c|c|c|c|c|c|}
\hline Characteristics & $\begin{array}{c}\text { Dysmenorrhea } \\
(n=74)\end{array}$ & $\begin{array}{c}\text { Normal } \\
(n=62)\end{array}$ & Total & p value & OR & $95 \% \mathrm{CI}$ \\
\hline \multicolumn{7}{|l|}{ Age } \\
\hline Mean & 20.54 & 20.6 & 20.57 & \multirow{2}{*}{0.56} & \multirow{2}{*}{-} & \multirow{2}{*}{-} \\
\hline Median & 20.5 & 21 & 21 & & & \\
\hline \multicolumn{7}{|l|}{ Regularity } \\
\hline Regular & $56(53 \%)$ & $50(47 \%)$ & $106(78 \%)$ & \multirow{2}{*}{0.49} & \multirow{2}{*}{0.75} & \multirow{2}{*}{$0.33-1.70$} \\
\hline No & $18(60 \%)$ & $12(40 \%)$ & $30(22 \%)$ & & & \\
\hline \multicolumn{7}{|l|}{ Menstrual cycle } \\
\hline$<25$ days & $8(57 \%)$ & $6(43 \%)$ & $14(10 \%)$ & \multirow{3}{*}{0.80} & 1 & 1 \\
\hline 25-32 days & $58(55 \%)$ & 47 (45\%) & $105(77 \%)$ & & 0.67 & $0.16-2.77$ \\
\hline$>32$ days & $8(47 \%)$ & $9(53 \%)$ & 17 (13\%) & & 0.72 & $0.26-2.01$ \\
\hline \multicolumn{7}{|l|}{ Duration } \\
\hline$<7$ days & $41(56 \%)$ & $32(44 \%)$ & $73(54 \%)$ & \multirow{2}{*}{0.66} & \multirow{2}{*}{1.17} & \multirow{2}{*}{$0.59-2.29$} \\
\hline $7-14$ days & $33(52 \%)$ & $30(48 \%)$ & $63(46 \%)$ & & & \\
\hline \multicolumn{7}{|l|}{ Amount of bleeding } \\
\hline$<3$ pads/day & $32(55 \%)$ & $26(45 \%)$ & $58(43 \%)$ & \multirow{2}{*}{0.88} & \multirow{2}{*}{1.06} & \multirow{2}{*}{$0.53-2.09$} \\
\hline 3-8 pads/day & $42(54 \%)$ & $36(46 \%)$ & $78(57 \%)$ & & & \\
\hline \multicolumn{7}{|c|}{ Gynecological problems } \\
\hline Yes & $2(40 \%)$ & $3(60 \%)$ & $5(4 \%)$ & \multirow{2}{*}{0.51} & \multirow{2}{*}{0.55} & \multirow{2}{*}{$0.08-3.38$} \\
\hline No & $72(55 \%)$ & $59(45 \%)$ & $131(96 \%)$ & & & \\
\hline \multicolumn{7}{|l|}{$\begin{array}{l}\text { Family history of } \\
\text { dysmenorrhea }\end{array}$} \\
\hline Yes & $45(66 \%)$ & $23(34 \%)$ & $68(50 \%)$ & \multirow{2}{*}{0.006} & \multirow{2}{*}{2.63} & \multirow{2}{*}{$1.31-5.27$} \\
\hline No & $29(43 \%)$ & $39(57 \%)$ & $68(50 \%)$ & & & \\
\hline \multicolumn{7}{|l|}{ Smoking habit } \\
\hline Yes & $2(100 \%)$ & $0(0 \%)$ & $2(2 \%)$ & \multirow{2}{*}{0.19} & \multirow{2}{*}{1.86} & \multirow{2}{*}{$1.59-2.18$} \\
\hline No & $72(54 \%)$ & $62(46 \%)$ & $134(98 \%)$ & & & \\
\hline Coffee consumption & & & & & & \\
\hline Yes & $24(49 \%)$ & $25(51 \%)$ & $49(36 \%)$ & 034 & 71 & 025112 \\
\hline No & $50(57 \%)$ & $37(43 \%)$ & 87 (64\%) & 0.04 & 0.11 & $0.53-1.45$ \\
\hline Alcohol consumption & & & & & & \\
\hline Yes & $2(50 \%)$ & $2(50 \%)$ & $4(3 \%)$ & 086 & 082 & $011-600$ \\
\hline No & $72(55 \%)$ & $60(45 \%)$ & $132(97 \%)$ & 0.00 & 0.03 & $0.11-0.09$ \\
\hline
\end{tabular}

except family history of dysmenorrhea (p 0.006). Lifestyle characteristics in the subjects also had no statistically significant differences.

Table 2 shows that among 136 female medical students were included in analysis, the overall proportion subjects with moderatesevere, and no-mild stress were $21 \%$ (28 subjects) and 79\% (108 subject), respectively.
The relationship between the severity of stress and the presence of last menstrual cramps was not statistically significant ( $p$ 0.745). The women with more severe stress had $54 \%$ probability to have dysmenorrhea in general population (Odd Ratio [OR] 1.15, 95\% Confidence Interval [CI] 0.5-2.66).

Table 3 shows that among 74 female medical 
Table 2 Relationship between the Severity of Psychological Stress and the Presence of Dysmenorrhea in Last Menstruation

\begin{tabular}{|c|c|c|c|c|c|c|c|}
\hline & & \multicolumn{2}{|c|}{ Last Menstrual Cramps } & \multirow{2}{*}{ Total } & \multirow{2}{*}{ p value } & \multirow{2}{*}{ OR } & \multirow{2}{*}{$95 \% \mathrm{CI}$} \\
\hline & & yes & no & & & & \\
\hline \multirow[t]{2}{*}{ Stress } & Moderate-severe & 16 & 12 & 28 & \multirow{2}{*}{0.745} & \multirow{2}{*}{1.15} & \multirow{2}{*}{$0.5-2.7$} \\
\hline & No-mild & 58 & 50 & 108 & & & \\
\hline Total & & 74 & 62 & 136 & & & \\
\hline
\end{tabular}

students with dysmenorrhea, the overall proportion subjects with moderate-severe and no-mild stress were $22 \%$ (16 subjects) and $78 \%$ (58 subjects). The relationship between the severity of stress and the severity of dysmenorrhea in last menstruation was statistically significant (p 0.033). The women with more severe stress had $79 \%$ probability to have more severe dysmenorrhea in population with dysmenorrhea (OR 3.69, 95\%CI 1.0612.8).

\section{Discussion}

This study revealed that the overall incidence of dysmenorrhea was $54.5 \%$ of 136 subjects with the mild, moderate, and severe dysmenorrhea that reached $12.1 \%, 36.5 \%$, and $51.4 \%$. It was similar to the previous study by Zukri, et al. ${ }^{6}$, in 2009 that reached $52.2 \%$ of 235 subjects. It seems that the medical student characteristics in Malaysia and Indonesia were similar. Among all factors assessed in this study, family history of dysmenorrhea had a strongest association with dysmenorrhea than other factors (OR 2.63, 95\%CI: 1.31-5.27). This study result is in the line with the previous study by Unsal et al. ${ }^{11}$ in 2010 that stated family history seems to be an important factor to affect women having dysmenorrhea (OR 20.7, 95\% CI: 11.537.4). A study by Tavallae, et al. ${ }^{5}$ in 2011 also found a relation between family history of dysmenorrhea and having dysmenorrhea (OR 2.60, 95\% CI 1.50-4.70).

The relationship between psychological stress and dysmenorrhea on last menstruation in this study showed no statistically significant result ( $p$ 0.745), but the women with more severe stress had $54 \%$ probability to have dysmenorrhea in general population (OR 1.15, 95\% CI 0.5-2.66). In addition, the relationship between the severity of stress and the severity of dysmenorrhea in last menstruation among those having dysmenorrhea was statistically significant ( $p$ 0.033), it also means that the women with more severe stress have $79 \%$ probability to have more severe dysmenorrhea in population with dysmenorrhea (OR 3.69, 95\%CI 1.06-12.8).

These results are consistent with the previous study by Wang, et al. ${ }^{7}$ that found higher risk of having dysmenorrhea in women with high stress compared to women with low stress (OR 10.4, 95\% CI: 4.9-22.3). In addition, Yamamoto et al. ${ }^{12}$ in their study, revealed that women having menstrual cramps have higher score of stress than those who do not have menstrual cramps (OR 1.02, 95\% CI 0.991.04). Moreover, a study by Nohara et al. ${ }^{13}$ showed a significant relation between high level stress and menstrual cramps (OR 1.46, 95\% CI: 1.13-1.87).

The previous observed study revealed that the mechanisms of psychological stress and dysmenorrhea relationship are not completely

Table 3 The Relationship between the Severity of Stress and the Severity of Dysmenorrhea in Last Menstruation among Subjects with Dysmenorrhea

\begin{tabular}{|c|c|c|c|c|c|c|c|}
\hline & & \multicolumn{2}{|c|}{ Last Menstrual Cramps } & \multirow[b]{2}{*}{ Total } & \multirow[b]{2}{*}{ p value } & \multirow[b]{2}{*}{$\mathbf{O R}$} & \multirow[b]{2}{*}{$95 \% \mathrm{CI}$} \\
\hline & & severe & $\begin{array}{c}\text { mild- } \\
\text { moderate }\end{array}$ & & & & \\
\hline \multirow[t]{2}{*}{ Stress } & Moderate-severe & 12 & 4 & 16 & \multirow{2}{*}{0.033} & \multirow{2}{*}{3.69} & \multirow{2}{*}{$1.06-12.8$} \\
\hline & No-mild & 26 & 32 & 58 & & & \\
\hline Total & & 38 & 36 & 74 & & & \\
\hline
\end{tabular}


understood. However, a person who is under stress undergoes a neuroendocrine response. Cortisol that is regulated by corticotrophin releasing hormone (CRH) as the major hypothalamic regulator stress response will mediate its secretion through pituitary adrenocorticotrophic hormone (ACTH); and progesterone that takes a part in follicular development may be disrupted due to follicular stimulating hormone and luteinizing hormone impairment. These conditions were assumed to play important roles in dysmenorrhea mechanisms that have been shown to influence both of prostaglandin PGE2 and PGF2 synthesis as well as their binding on myometrial receptors that will affect uterine muscle and vascular tone., ${ }^{3,7}$

This study might be the first study that associates with psychological stress and dysmenorrhea specifically and used more validated questionnaire for assessed variable. Previously, the study used self-perception to assess whether they felt menstrual cramps or not and in a high or lower stress. In addition, the subjects were almost remarkably homogenous in their age and nulliparous that made the results were not influenced by parity and wide range of age.

There were some limitations in this study. First, it was a cross-sectional study that could not determine the causality among variable, but it only could determine the relationship and its strength. The seconds, since this study was a self-assessment questionnaire, it might result an underreporting condition. Lastly, it was conducted in a single university with few subjects, therefore, the sample might not be representative of all Indonesian female medical students.

For further studies, it is suggested to arrange larger study that more represents the real population. In addition, since there was a positive relationship between psychological stress and severity of dysmenorrhea, a study associating with stress management as a method to prevent more severe conditions among dysmenorrhea women might be conducted in the next studies.

In conclusion, the menstrual profile that has the strongest relation with dysmenorrhea is the family history of dysmenorrhea. There is also a positive relationship between the severity of stress and dysmenorrhea in last menstruation among those having dysmenorrhea. So, the stress management might be arranged to reduce the stress level, prevent more severe dysmenorrhea, and increase the productivity among women with dysmenorrhea.

\section{References}

1. Lentz GM, Lobo RA, Gershenson DM, Katz VL. Comprehensive Gynecology. Philadelphia, PA: Mosby Elsevier; 2012.

2. Patel V, Tanksale V, Sahasrabhojanee M, Gupte S, Nevrekar P. The burden and determinants of dysmenorrhoea: a population-based survey of 2262 women in Goa, India. BJOG. 2006;113(4):453-63.

3. Ju H, Jones M, Mishra G. The prevalence and risk factors of dismenorrhea. Epidemiol Rev. 2013:36(10):104-13.

4. Ohde S, Tokuda Y, Takahashi O, Yanai H, Hinohara S, Fukui T. Dysmenorrhea among Japanese women. Int J Gynaecol Obstet. 2008;100(1):13-7.

5. Tavallaee M, Joffres MR, Corber SJ, Corber SJ, Bayanzadeh M, Rad MM. The prevalence of menstrual pain and associated risk factors among Iranian women. J Obstet Gynaecol Res. 2011;37(5):442-51.

6. Zukri SM, Naing L, Hamzah TNT, Husain NHN. Primary dysmenorrhea among Medical and Dental University students in Kelantan: prevalence and associated factors. International Medical Journal. 2009:16(2):93-9.

7. Wang L, Wang $\mathrm{X}$, Wang $\mathrm{W}$, Chen $\mathrm{C}$, Ronnennberg A G, Guang W, et al. Stress and dysmenorrhoea: a population based prospective study. Occup Environ Med. 2004:61:1021-6.

8. Damanik ED. The measurement of reliability, validity, items analysis and normative data of depression anxiety stress scale (DASS) [thesis]. Jakarta: Faculty of Psychology: Universitas Indonesia, Indonesia; 2011.p. 60.

9. Hjermstad MJ, Fayers PM, Haugen DF, Caraceni A, Hanks GW, Loge JH, et al. Studies comparing numerical rating scales, verbal rating scales, and visual analogue scales for assessment of pain intensity in adults: a systematic literature review. J Pain Symptom Manage. 2011;41(6):107393.

10. Garra G, Singer AJ, Domingo A, ThodeJr HC. The Wong-Baker pain FACES scale measures pain, not fear. Pediatr Emerg Care. 2013;29(1):17-20.

11. Unsal A, Ayranci U, Tozun M, Arslan G, Calik E. Prevalence of dysmenorrhea and its effect on quality of life among a group of female university students. Ups J Med Sci. 2010:115(1):138-45.

12. Yamamoto $\mathrm{K}$, Okazaki A, Sakamoto $\mathrm{Y}$, Funatsu M. The relationship between 
premenstrual symptoms, menstrual pain, irregular menstrual cycles, and psychosocial stress among Japanese College Students. J Physiol Anthropol. 2009.28(1):129-36.
13. Nohara M, Momoeda M, Kubota T, Nakabayashi M. Menstrual cycle and menstrual pain problems and related risk factors among Japanese female workers. Ind Health. 2011;49(2):228-34. 